\title{
Synergistic Effects Under Ion-Beam Modification of Metals
}

\author{
Anatoly Borisov ${ }^{1,2, *}$, Boris Krit ${ }^{1,2}$, Igor Suminov ${ }^{2}$, Mikhail Ovchinnikov ${ }^{3}$ and Sergey Tikhonov ${ }^{1}$ \\ ${ }^{1}$ Moscow Aviation Institute (National Research University), RU-125993 Moscow, Russia \\ ${ }^{2}$ Moscow State Technological University "STANKIN", RU-127994 Moscow, Russia \\ ${ }^{3}$ Skobeltsyn Institute of Nuclear Physics, Moscow State University, RU-119991 Moscow, Russia
}

\begin{abstract}
The combined effect of ion and laser beams on physical and mechanical properties of metal and alloy surfaces has been studied. The technique of determining the main parameters of polyenergetic ion implantation using a vacuum-arc ion source is proposed and evaluated. It is found that treatment with titanium ions and the subsequent laser thermal hardening increase microhardness of steel 45 and U8 up to 6 times.
\end{abstract}

\section{Introduction}

The material processing with concentrated energy flows allows changing physical and chemical surface properties in a wide range. The ion and laser beams are extensively used to modify the surface of materials. In the context of ion processing, in particular ion implantation, surface properties of materials are modified as a result of the implantation of high-energy ions causing changes in composition of elements (doping) and structure-phase states of subsurface layers. It is possible to change directly such properties of materials as microhardness, wear resistance, corrosion resistance, heat resistance as well as friction, optical, magnetic, emission properties, etc. by using ion implantation.

The progress of laser techniques has caused wide application of lasers for the materials processing. One of the perspective directions of laser technology from the point of view of materials properties modification is surface laser hardening in which the structure-phase state of subsurface layers is changed by pulse heat treatment. The prospects of such technology are determined by the possibility to create various new surface structures with improved physical, chemical and mechanical properties in sufficiently thick layers using relatively simple equipment.

The analysis of different technological processes showed that the efficiency of laser processing methods could be significantly increased by creating the laser combined methods based on the combination of laser sources with other sources of synergistic effect on materials.

For the ion processing of structural materials, a significant change in properties occurs when the concentration of dopant reaches a density comparable to the density of a solid material. For this reason, the required irradiation fluences are about $10^{16}-10^{18} \mathrm{~cm}^{-2}$ (high-dose range). For the high-dose ion implantation
(HDII) the ions with the energy, in which their stopping range in the material exceeds the thickness of the sputtered layer, are used. The depth of implantation for the ions with energies $\sim 10^{4}-10^{6} \mathrm{eV}$ are $0,1-1 \mu \mathrm{m}[1,2]$.

HDII can be considered as complex chemical, thermal and mechanical impact on materials. HDII has several advantages over other methods of material processing (e.g., vacuum deposition).

Nowadays, works on modification of properties of structural materials by ion-plasma flows which allow to change physico-mechanical, electrophysical, electrochemical properties of a surface layer of materials and work-pieces from them are intensively developing [2]. In these works the requirements to monoenergeticity and purity of the ion beam are reduced through the necessity to increase the productivity (processing dose $10^{16}-10^{18} \mathrm{~cm}^{-2}$ ) and the area of the processed work-pieces (respectively, ion beam cross section) up to $10^{2}-10^{3} \mathrm{~cm}^{2}$.

The non-separation industrial ion implanters, with the vacuum-arc pulsed metal ion sources of MEVVA type (metal vapor vacuum arc) have high productivity [2,3]. It is based on a complex composition of the ion beam both for ion charge and energy. The energy of single-charged ions is determined by the accelerating voltage, the energy of multi-charged ions is a multiple of the energy of single-charged ions, therefore, this ion implantation is polyenergetic.

The present work is aimed to study the complex (synergistic) effect on the microhardness of the surface of structural and instrumental steels of polyenergetic metal ion beams of, produced using a vacuum-arc source, and laser thermal hardening.

\section{Experimental techniques}

The non-separation ion implanter with pulse vacuum-arc ion source used in the work is illustrated by the scheme shown in Fig. 1.

Corresponding author: anatoly_borisov@mail.ru 


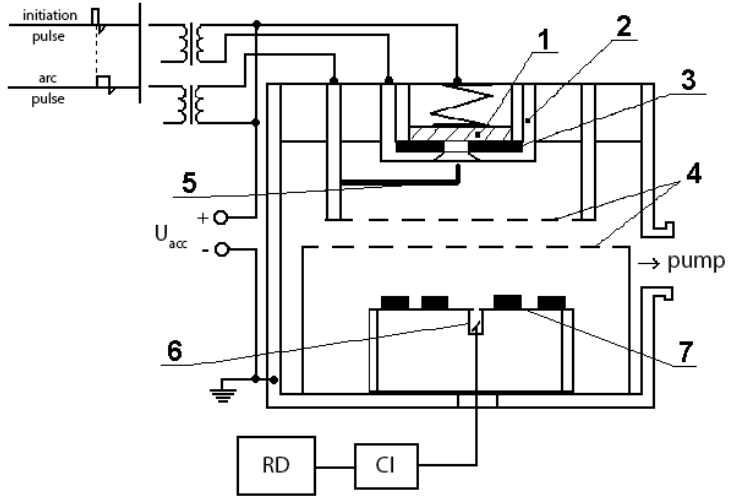

Fig. 1. Scheme of the implantation installation with pulse vacuum-arc ion source [4].

Arc ignition is initiated by electric breakdown of ceramic ring insulator - 3 between replaceable watercooled cathode - 1 with diameter $10 \mathrm{~mm}$ and ignition electrode -2 . The arc discharge is supported by a pulse power supply providing about 300 A current of the arc during $300 \mu \mathrm{s}$. The acceleration of ions of cathode material, generated by vacuum arc, takes place in the electrostatic field between tungsten meshes of ionoptical system - 4. Application of rod anode insert - 5 allows more than 3 times increase in current of extractable ions due to change of indicator of ions output from cathode. The dosimetry of ion irradiation of samples, installed on the object table - 7 , is performed using the Faraday cylinder - 6 and current integrator (CI). The current integrator converts the charge, which passed through the Faraday cylinder, into the frequency of pulse repetition, which is then summed up by recalculating device (RD). The implanter allows obtaining wide aperture round cross-section (diameter of output aperture $30 \mathrm{~cm}$ ) metal ion beams with current 0.1 $1 \mathrm{~A}$, pulse duration $\sim 300 \mu \mathrm{s}$, pulse repetition frequency up to $50 \mathrm{~Hz}$. Maximum value of accelerating voltage $U_{\text {acc }}=50 \mathrm{kV}$.

The conditions of irradiation of steel samples (St. 45, 40X13, U8) and graphite selected as a witness - sample of polyenergetic ion implantation are presented in Table 1.

Table 1. Ion implantation parameters and Rutherford spectrometry results for graphite ion implantation

\begin{tabular}{|c|c|c|c|c|c|c|c|}
\hline \multirow[t]{2}{*}{$\begin{array}{l}\text { Cathode } \\
\text { material }\end{array}$} & \multirow{2}{*}{$\begin{array}{c}\text { Ion charge } \\
\text { concentrations } \\
\text { in the beam } \\
q=1^{+}, 2^{+}, 3^{+} \\
4^{+}, 5^{+}, \%\end{array}$} & \multirow[t]{2}{*}{$q_{\text {mean }}$} & \multirow[t]{2}{*}{$\begin{array}{l}U_{\mathrm{acc}} \\
\mathrm{kV}\end{array}$} & \multirow[t]{2}{*}{$\begin{array}{c}F \\
10^{17} \\
\mathrm{~cm}^{-2}\end{array}$} & \multicolumn{3}{|c|}{$\begin{array}{c}\text { Rutherford } \\
\text { backscattering } \\
\text { spectroscopy and } \\
\text { simulation results }\end{array}$} \\
\hline & & & & & $\begin{array}{c}D_{\text {Ruth }}, 10^{17} \\
\mathrm{~cm}^{-2}\end{array}$ & $S$ & $\begin{array}{l}D_{\text {sim }}, \\
10^{17} \\
\mathrm{~cm}^{2}\end{array}$ \\
\hline $\mathrm{Ti}$ & $11,75,14$ & 2,1 & 35 & 6,0 & 1,7 & 1,55 & 3,0 \\
\hline $\mathrm{Al}$ & $38,51,11$ & 1,7 & 35 & 0,4 & 0,73 & 0,67 & 0,4 \\
\hline $\mathrm{Nb}$ & $1,24,51,22,2$ & 3,0 & 35 & 3,0 & 0,39 & 3,24 & 1,34 \\
\hline $\mathrm{Cu}$ & $16,63,20,1$ & 2,0 & 35 & 0,6 & 0,6 & 0,99 & 0,59 \\
\hline $\mathrm{Cr}$ & $10,68,21,1$ & 2,1 & 35 & 4,2 & 2,5 & 1,75 & 3,0 \\
\hline $\mathrm{Pb}$ & 36,64 & 1,6 & 20 & 0,3 & 0,2 & 3,07 & 0,24 \\
\hline
\end{tabular}

The element composition of the implanted layer was analyzed using the Rutherford backscattering spectroscopy (RBS) of a $2 \mathrm{MeV}$ helium ion beam obtained at the electrostatic ion accelerator of the SINP MSU using the same method as in $[4,5]$.

Laser processing was carried out on the laser installation "Kvant-18" with pulse duration $8 \mathrm{~ms}$, pulse overlapping factor 0.7 and laser beam power density $2 \mathrm{~W} / \mathrm{cm}^{2}$, which preserved the surface microgeometry and did not melt it.

Microhardness was measured according to Vickers on the multipurpose measuring complex "METALPLAN" by LEITZ at the indenter loads from 50 to $100 \mathrm{~g}$.

\section{Results and discussion}

The experimental data obtained in the present work on polyenergetic high-fluence implantation (Table 1) were considered in the framework of the analytical model of high-fluence ion implantation, which takes into account one dominant factor - sputtering of the target by ion bombardment [5].

Under polyenergetic implantation, when the energy of ions is determined by their charge $q=1,2,3, \ldots$, the partial concentration cross sections of ions $N_{\mathrm{q}}(x)$ in the target can be described in a form:

$$
N_{q}(x)=\frac{N_{O} \cdot n_{q}}{2 S}\left[\operatorname{erf}\left(\frac{x+\frac{F}{N_{O}} S-R_{q}}{\sqrt{2} \cdot \Delta R_{q}}\right)-\operatorname{erf}\left(\frac{x-R_{q}}{\sqrt{2} \cdot \Delta R_{q}}\right)\right],
$$

where, $N_{0}$ - concentration of target atoms; $n_{\mathrm{q}}$ - relative concentration of ions with charge $q$ in the beam; $R_{\mathrm{q}}$ and $\Delta R_{\mathrm{q}}$ - projected range and mean square straggling of projected range of ions with charge $q$, respectively; $F$ fluence of implantation. The sputtering yield for polyenergetic ion irradiation:

$$
S=\sum_{q=1}^{n} S_{q} n_{q}
$$

where $S_{\mathrm{q}}$ is the sputtering yield of the target by ions with charge $q$. Values of $S_{\mathrm{q}}$ were calculated using SRIM software.

Total concentration cross section of implanted atoms:

$$
N(x)=\sum_{q=1}^{n} N_{q}(x)
$$

Integrated dose of implantation:

$$
D(x)=\int N(x) d x
$$

An example of calculations of partial and total concentration cross sections is shown in Figs. 2, 3. The total concentration cross section is significantly determined by the charge composition of the ion beam (Fig. 2).

The analysis of RBS spectra in case of irradiation with fluence above $10^{17} \mathrm{~cm}^{-2}$ revealed the presence of 
impurities implanted in the samples. The detected impurities (not more than $5 \%$ of the implanted element) such as tungsten, molybdenum, aluminum are the elements of the ion source. In the case of titanium ion implantation, oxygen was also found, the atomic concentration of which is comparable to that of implanted titanium, which indicates the possible formation of titanium oxides such as $\mathrm{TiO}$ and $\mathrm{Ti}_{2} \mathrm{O}_{3}$ on the sample surface.

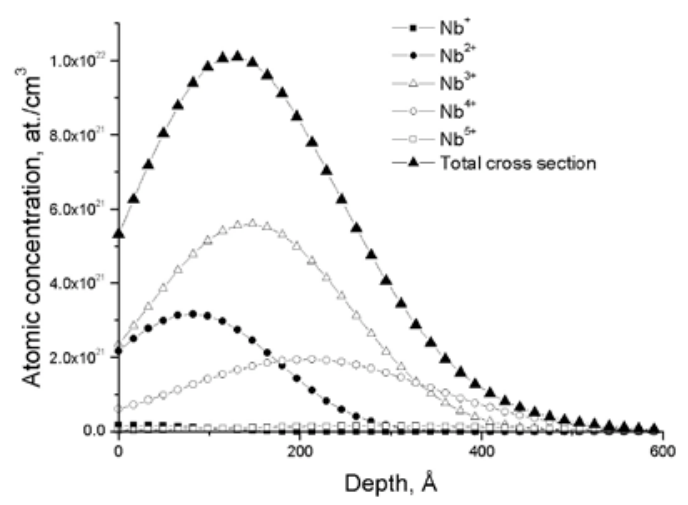

Fig. 2. Partial and total concentration cross sections of niobium implanted in 40X13 steel under irradiation fluence $F=3 \cdot 10^{17} \mathrm{~cm}^{-2}$.

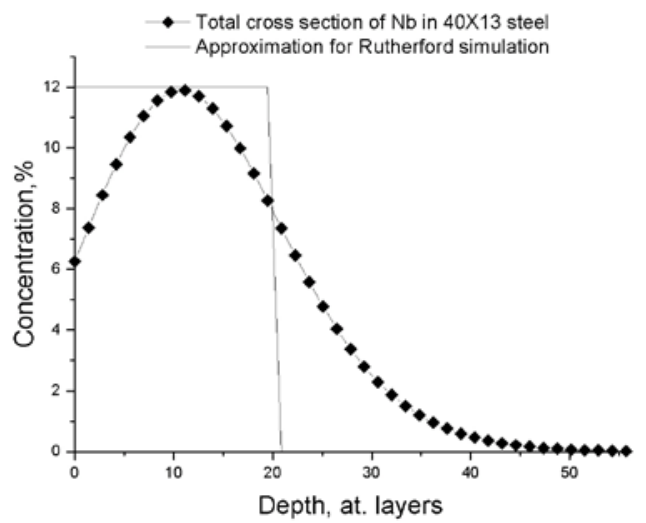

Fig. 3. Total cross section of $\mathrm{Nb}$ in steel $40 \mathrm{X} 13$ and its approximation for RBS simulation.

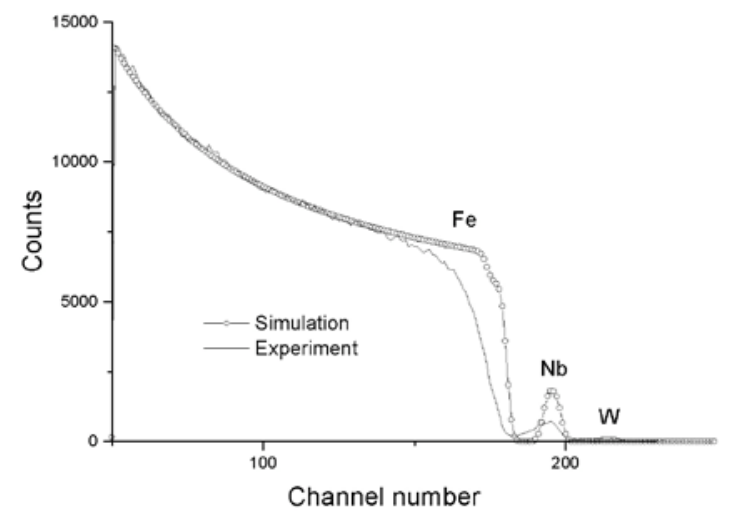

Fig. 4. Comparison of calculated and experimental RBS spectrum for $\mathrm{Nb}$ implanted in steel 40X13.
Some differences in the peak shape for $\mathrm{Nb}$ implanted in 40X13 steel (Fig. 4) may also be related to the selected approximation of its simulated cross section (Fig. 3).

Since the concentration profile of the implanted element is significantly influenced by the ion charge, which determines the ion stopping range in the material and sputtering of the surface, as well as their partial composition in the beam, on which the overall profile depends, the results of the difference between the simulation of polyenergetic ion implantation and the experiment (Table 1) can be associated with the following factors:

1. The difference of the used data in [3] by the charge composition of $n_{\mathrm{q}}$ ion beams produced by MEVVA type vacuum-arc ion sources (Table 1 ) with the corresponding composition for the implanter ion source.

2. The concentration of the implanted element depends on the sputtering yield $S$, which is a function of ion charge $q$. The value of projected run, on the other hand, depends on the energy of ions, which is a product of charge $q$ and accelerating voltage $U_{\text {acc. }}$. Thus, because of this incompatibility of charge data, it is unknown what effect on the difference between the calculated and experimental RBS spectra was greater: sputtering yield or projected range.

3. In this model, the values of projected ranges, stragglings and sputtering yield calculated with the SRIM program were used. This program simulates sputtering in linear cascade mode. In our case, heavy ions are implanted and, consequently, a thermal peak mode is possible, which is not considered in the SRIM program.

4. The presence of impurities may also affect the concentration cross section of the implanted dopant, since the presence of impurities is not taken into account in the simulation of the implantation process.

Table 2 shows the results of microhardness measurement at loading on the indenter $50 \mathrm{~g}$ and parameters of ion implantation for steels $40 \mathrm{X} 13$, st. 45 , U8.

Table 2. Ion implantation parameters and microhardness of steels.

\begin{tabular}{|c|c|c|c|c|c|c|c|}
\hline \multirow[t]{2}{*}{ \# } & \multirow[t]{2}{*}{ Io } & \multirow{2}{*}{$\begin{array}{c}U_{\mathrm{acc}}, \\
\mathrm{kV}\end{array}$} & \multirow{2}{*}{$\begin{array}{c}F, 10^{17} \\
\mathrm{~cm}^{-2}\end{array}$} & \multirow{2}{*}{$\begin{array}{l}\text { Irradiation } \\
\text { time, min }\end{array}$} & \multicolumn{3}{|c|}{ Microhardness } \\
\hline & & & & & $40 \times 13$ & st. 45 & U8 \\
\hline 1 & & Witl & out irradi & iation & $301 \pm 4$ & $105 \pm 4$ & $104 \pm 3$ \\
\hline 2 & $\mathrm{Cu}$ & 35 & $5.8 \cdot 10^{16}$ & 110 & $380 \pm 5$ & $126 \pm 3$ & $151 \pm 3$ \\
\hline 3 & $\mathrm{~Pb}$ & 20 & $3 \cdot 10^{16}$ & 52 & $393 \pm 6$ & $113 \pm 3$ & $154 \pm 6$ \\
\hline 4 & $\mathrm{Al}$ & 35 & $4 \cdot 10^{16}$ & 70 & $389 \pm 5$ & $123 \pm 5$ & $129 \pm 4$ \\
\hline 5 & $\mathrm{Ti}$ & 35 & $2 \cdot 10^{17}$ & 135 & $537 \pm 10$ & $159 \pm 6$ & $183 \pm 5$ \\
\hline 6 & $\mathrm{Nb}$ & 35 & $3 \cdot 10^{17}$ & 162 & $546 \pm 9$ & $158 \pm 5$ & $175 \pm 6$ \\
\hline
\end{tabular}

The depth of ion stopping range, calculated using the SRIM program, in steel does not exceed 0.1 micrometers, and the indenter bulge depth is one micrometer. The effect of increasing microhardness in such cases is usually associated with the long-range effects of ion implantation [6]. 
One of the mechanisms of such effects is the radiation stimulated diffusion of point defects and formation of dislocation loops, which cause the lattice distortion and hardening of the material $[1,6]$. The flow of interstitial atoms in depth of sample can cause dislocation creep. Thus, radiation defects and elastic stresses can penetrate to much greater depths than the stopping range of ions, and have a significant impact on the properties of the surface layers.

It is found that significant increase in microhardness of the surface is observed at ion bombardment with titanium and niobium ions.

After ion implantation, laser thermal hardening of samples from structural and instrumental steels was carried out at "Kvant-18" installation. Results of microhardness measurement are shown in fig. 5 .

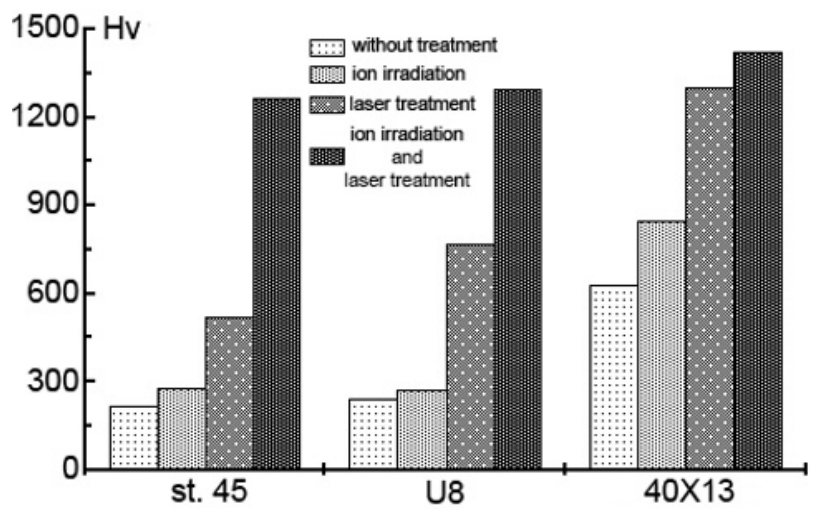

Fig. 5. Influence of titanium ion implantation and laser treatment on microhardness of 45, U8, 40X13 steels

A dramatic increase in microhardness of steels under combined ion-laser treatment can be explained by the influence of radiation defects formed by ions and the implanted impurities on the subsequent formation of the hardening structure during laser thermal hardening, changes in the microstructure of the surface and, in particular, an increase in the dispersion of martensite.

\section{Conclusions}

Possibilities of analytical methods of determination of the main parameters of polyenergetic ion implantation with the use of vacuum-arc ion source have been analyzed using Rutherford backscattering spectroscopy of implanted samples.

The impact of ion implantation on surface hardening of structural and instrumental steels was studied. It is found out that ion implantation increases microhardness of the steel surface by $20-80 \%$ depending on the type of ions. The best results of microhardness increase of steels U8, 45 and $40 \mathrm{X} 13$ in 1,5-1,8 times were obtained by treatment with titanium and niobium ions at the irradiation fluences $\sim 10^{17} \mathrm{~cm}^{-2}$.

The effects of laser heat treatment on microhardness of steels have been studied. The obtained results show an increase in microhardness of U8, 45 and 20X13 steels surface by 1.3-2.5 times.

The results show that combined ion-laser treatment of steels is promising. It was found that treatment with titanium ions and subsequent laser thermal hardening increase microhardness of 45 and U8 steel up to 6 times, and thermally pre-hardened steel $40 \mathrm{X} 13$ - more than 2 times.

\section{Acknowledgments}

The study was carried out with the financial support of the Russian Foundation for Basic Research within the framework of scientific project No. 21-79-30058.

\section{References}

1. K.J. Kirkby, R.P. Webb, Encylopedia of Nanoscience and Technology, 4, 1-11 (2004)

2. J.M. Poate, G. Foti, D.C. Jacobson, Surface Modification and Alloying by Laser, Ion, and Electron Beams (Springer Science Business Media, New York, 1983)

3. Ian G. Brown, The Physics and Technology of Ion Sources (John Wiley \& Sons, University of California. Berkeley, California, 1989)

4. A.M. Borisov, B.L. Krit, S.A. Tikhonov, I.V. Suminov, V.S. Kulikauskas, V.G. Sukharev, Bull. Russ. Acad. Sci. Phys., 64, 4 (2000)

5. A.M. Borisov, N.V. Borodulina, B.L. Krit, S.A. Tikhonov, J. Appl. Phys., 1, 89-93 (2004)

6. R. Smith, M. Jakas, D. Ashworth, R. Oven, M. Bowyer, I. Chakarov, R.P. Webb, Atomic \& Ion Collisions in Solids and at Surfaces (Cambridge University Press, Cambridge, 1997) 